ImplantaCión de ECTS en Administración y Dirección de Empresas: Comparacion de Resultados entre Materias de Primer y Últtimo Curso

\author{
M. Dioni Elche Hortelano * \\ Pedro Manuel Garcia Villaverde \\ Ricardo Martinez Cañas \\ Gloria Parra Requena \\ Pablo Ruiz Palomino
}

RESUMEN:

Este artículo presenta los resultados de un estudio empírico en el cual se comparan los resultados obtenidos entre asignaturas iniciales y' finales de la Licenciatura de Administración y Dirección de Empresas; utilizando metodologias de aprendizaje activo para la implantación del ECTS Los aspectos analizados son el diseño y utilidad de las Guias Docentes, el nivel de satisfacción con las técnicas docentes y el desarrollo de las asignaturas, la valoración de la mejora de competencias, el nivel de esfuerzo desarrollado y la satisfacción con los resultados obtenidos. El análisis realizado permite establecer un perfil diferenciado entre los alumnos de primer y último curso en la forma de percibir y afrontar la implantación del ECTS, asi como en la satisfacción con los resultados obtenidos.

\title{
Palabras Clave:
}

Créditos ECTS. Aprendizaje activo. Satisfacción de los estudiantes. Mejora de las competencias.

- Departamento de Administración de Empresas. Facultad de Ciencias Sociales. Universidad de CastillaLa Mancha • Dioni.Elche@uclm.es 
ABSTRACT:

This paper presents findings from an empirical study which compares students' performance among courses at the beginning and the end of Business Studies and Economics. It uses active learning methodology for the implantation of ECTS (European Credits Transfer System). The factors under analysis are design and usefulness of the students' guide; satisfaction with teaching techniques and development of subjects; evaluation of competence improvement; effort level and overall students' satisfaction. This analysis allows establishing a different profile between students of the first and the last course in relation to bow they perceive and confront the introduction of ECTS, as well as their satisfaction with overall performance

KEY WORDS:

ECTS. Active learning methodology. Satisfaction of students. Competences improvement.

\section{INTRODUCCIÓN}

En los últimos años, la mayor parte de las universidades españolas han abordado una cierta aproximación al Espacio Europeo de Enseñanza Superior ${ }^{1}$. Una de las iniciativas que se está desarrollando en varias universidades es la aplicación de planes de entrenamiento para la implantación del ECTS (European Credit Transfer System). Para potenciar este proceso en la Universidad de Castilla-La Mancha se ha creado la Unidad de Innovación y Calidad Educativa, que tiene como misión coordinar actividades dirigidas a apoyar la docencia de calidad, estimular, apoyar y dar visibilidad a los esfuerzos de mejora educativa; impulsar la comunicación entre equipos docentes con el entorno y proporcionar formación técnica ${ }^{1}$. En diversos centros y titulaciones se plantea un debate sobre la conveniencia de desarrollar los programas de entrenamiento para el conjunto de la titulación o de manera gradual y, en este caso, empezando por los cursos inferiores o superiores. En este contexto consideramos interesante contrastar la forma de percibir y afrontar los planes de entrenamiento de la implantación del ECTS, así como la satisfacción con los resultados obtenidos entre los alumnos que se incorporan a la Licenciatura en Administración de Empresas -primer curso- y los que están próximos a la finalización de sus estudios -quinto curso-.

1. La Facultad de Ciencias Sociales de Cuenca tiene un papel activo en el desarrollo de planes de entrenamiento del ECTS. 
Los argumentos anteriores justifican que hayamos realizado este estudio que se enmarcar en un proyecto de innovación docente más amplio. El principal objetivo que nos proponemos en este trabajo es analizar de forma comparativa los resultados obtenidos de la aplicación de métodos de aprendizaje activo para la implantación del ECTS en asignaturas iniciales y finales de la Licenciatura de Administración y Dirección de Empresas.

Este trabajo se estructura en ocho apartados. Después de esta introducción, explicamos brevemente la génesis del EEES. Posteriormente, contextualizamos la experiencia realizada con alumnos de Administración y Dirección de Empresas en la Facultad de Ciencias Sociales, en el punto tres. En el siguiente describimos las asignaturas que se han analizado. En el quinto apartado planteamos el diseño de la investigación. En el sexto se presentan los resultados obtenidos de los análisis estadísticos realizados y en el séptimo, se exponen las principales conclusiones y propuestas extraídas de este estudio; por último, se recogen las referencias bibliográficas más relevantes.

\section{HACIA UN SISTEMA EUROPEO DE ENSEÑANZA SUPERIOR}

Las Universidades europeas se encuentran inmersas en un proceso de cambio que se inició con la Declaración de la Soborna (1998) y Bolonia (1999) y que dio origen a la creación del Espacio Europeo de Enseñanza Superior (EEES), cuyo principal objetivo consiste en la armonización de los estudios superiores en el horizonte temporal del 2010. La organización del EEES debe desarrollarse a partir de los principios de calidad, movilidad, diversidad, competitividad y orientación. Y con la finalidad de lograr básicamente dos objetivos estratégicos, por un lado, incrementar el empleo en la Unión Europea y, por otro lado, convertir el sistema europeo de enseñanza superior en centro de atracción para profesores, investigadores y estudiantes a nivel mundial.

En realidad, el trasfondo que hay en el acuerdo de Bolonia es que el mercado de trabajo se está redimensionando más allá de los límites nacionales; además de que se está incrementando la demanda de formación cualificada a los trabajadores. En este sentido, existe una demanda cada vez mayor de competencias profesionales, así como de un mayor compromiso social, una mayor responsabilidad profesional y una mayor autonomía. En este contexto aparecen el ECTS como medida de la carga de trabajo del alumno y con el fin de facilitar la movilidad de estudiantes a nivel internacional, ya que es un sistema que permite la transferencia y acumulación de créditos entre diferentes titulaciones y distintos países. La implantación del ECTS en el sistema educativo universitario supone una redefinición del actual concepto de crédito, que además implica la necesidad de un cambio en la metodología docente para convertir nuestro sistema universitario en un sistema que sea homologable.

El debate abierto por la Comisión de las Comunidades Europeas (2003) está centrado en el papel que las universidades están jugando en la sociedad del conocimiento, 
y sobre las condiciones en las que podrán desempeñar efectivamente su papel. La economía y la sociedad del conocimiento nacen de la combinación de cuatro elementos interdependientes: a) la producción del conocimiento, esencialmente por medio de la investigación científica, b) la transmisión del conocimiento mediante la educación y la formación, c) la difusión del conocimiento a través de las tecnologías de la información y la comunicación y d) la explotación del conocimiento a través de la innovación tecnológica. En este escenario, el sistema universitario español debe aprovechar esta revolución del ámbito educativo para cambiar su estructura y funcionamiento, ya que los métodos actuales están basados en los principios iniciales que regían la universidad en el S. XIV. Por lo tanto, este proceso de convergencia europeo es una oportunidad para adecuar la universidad a las necesidades de la sociedad actual, que está demandando profesionales competentes y comprometidos socialmente. Para ello es absolutamente necesario un cambio en el sistema formativo que se focalice en el desarrollo de competencias profesionales, es decir, se requiere un sistema universitario profesionalizante. Éste es uno de los retos más importantes que se plantea en el informe sobre Gestión del Conocimiento en la Sociedad del Aprendizaje (2000) de la OCDE, al que tienen que hacer frente la formación y el aprendizaje de hoy en día.

Esta nueva realidad social exige una nueva metodología de enseñanza en el ámbito universitario, la implantación de una formación por competencias requiere que tanto los profesores como los alumnos cambien sus prácticas y rutinas; más aún, deben cambiar también de mentalidad porque todo este proceso está suponiendo una revolución total del concepto de enseñanza en el sentido más amplio del término.

Dentro del Documento-Marco sobre Integración del Sistema Universitario Español en el EEES (MECD, 2003), los nuevos objetivos formativos de las enseñanzas oficiales están orientados a la profesionalización del alumnado y para ello es necesario formar en competencias desarrolladas de manera específica dependiendo de la cada titulación. En este nuevo contexto los tradicionales programas educativos por objetivos deben ser cambiados por programas basados en competencias. Esto conlleva obligatoriamente la incorporación de cambios en la metodología educativa. Es necesario romper con el sistema de educación tradicional -centrado en el profesor como director cuyo objetivo es la enseñanza mediante métodos repetitivos y estudio individual- para impulsar el aprendizaje activo centrado en el alumno, desarrollado de forma grupal a través de un proceso autoiniciado, a partir del análisis, síntesis y comprensión de los contenidos.

La sociedad actual está inmersa en una revolución irreversible debido a la globalización, la informatización, la desintermediación económica y la intangibilización (Stewart, 1997). Estos cambios socioeconómicos conllevan la rápida obsolescencia de los conocimientos existentes. El conocimiento, hoy en día, crece de forma exponencial y, por eso, se hace necesario el aprendizaje a lo largo de toda la vida. Ante esta situación, resulta más efectivo "aprender a aprender" para conseguir de este modo y mediante la autoformación, una formación continuada en el tiempo. Este es uno de los retos más importantes 
planteados en el informe sobre Gestión del Conocimiento en la Sociedad del Aprendizaje (2000) de la OCDE y al que tienen que hacer frente la formación y el aprendizaje que se demandan actualmente. El sistema educativo debe promover la utilización de métodos formativos que permitan la construcción de conocimiento a través del contenido. Para ello se deben modificar y orientar la metodología educativa hacia el aprendizaje, que se ha convertido en el principal objetivo de la enseñanza actual.

Partiendo de esta nueva realidad, en la que el conocimiento se construye mediante el aprendizaje continuado; aprender mediante un proceso de adquisición, comprensión, aplicación, análisis, síntesis y evaluación del conocimiento (Bloom, 1956) es más eficaz que la obtención de información simplemente a través de la lectura reiterativa y la memorización de contenidos. Los principios que rigen el aprendizaje son esencialmente la construcción, elaboración y organización del conocimiento, activando e integrando el conocimiento previo existente. Además, la contextualización de dicho aprendizaje es el elemento clave en este proceso porque estimula la generación de conocimiento, ya que al relacionar los conocimientos existentes con los nuevos facilita su recuerdo y asimilación.

\section{CONTEXTO DE APLICACIÓN}

Esta investigación se enmarca en el programa que la Facultad de Ciencias Sociales de Cuenca está desarrollando para la adaptación de sus titulaciones al Espacio Europeo de Enseñanza Superior (EEES) y dentro de las políticas de innovación educativa de la Universidad de Castilla-La Mancha. Para ello, se ha puesto en marcha en el Centro un proyecto para el desarrollo de la implantación de los créditos ECTS que se está realizando de forma gradual. De modo que en el curso pasado se puso en marcha este sistema en todas las asignaturas de primer curso; en el curso actual, se han incluido en el proyecto las asignaturas de segundo curso. No obstante, con carácter voluntario, se están llevando a cabo experiencias con metodologías de aprendizaje activo, dentro de este programa, en cursos superiores de las titulaciones. De hecho, de las dos asignaturas analizadas en este trabajo, una de ellas, Introducción a la Dirección de Empresas (IDE), se imparte en primer curso y, por tanto, está incluida en el proyecto piloto de la Facultad, mientas que la otra, Dirección Estratégica de Empresas (DEE), es de quinto curso, de modo que en este caso estamos aplicando los métodos de aprendizaje activo de manera voluntaria. En ambos casos, obligatoriamente se debe elaborar una guía docente de la asignatura, en la que se recoge de manera detalla todos los pasos que se siguen en el desarrollo del plan de aprendizaje: descripción de la asignatura, objetivos generales, volumen de trabajo, contenidos, competencias genéricas y destrezas, temario y planificación temporal, cronograma, bibliografia, metodologia empleada, sistema de evaluación del aprendizaje y evaluación del profesorado. 


\section{LAS ASIGNATURAS ANALIZADAS}

El contraste se ha realizado entre las asignaturas de Introducción a la Dirección de Empresas y Dirección Estratégica de la Empresa. La orientación de los objetivos generales ${ }^{2}$, la metodología ${ }^{3}$ y el sistema de evaluación -anexo 1- es similar para ambas materias.

\subsection{Introducción a la Dirección de Empresas}

Esta asignatura proporciona a los alumnos una aproximación a la Dirección de Empresas. Dentro del plan de estudios actual se encuadra en el primer semestre del primer curso de la Licenciatura de Adpministración y Dirección de Empresas. Con esta asignatura se pretende aportar a los alumnos un marco conceptual básico que les permita comprender qué es una empresa, su evolución histórica y sus principales elementos, así como el papel que ésta juega en la economía de mercado y las relaciones que mantiene con el resto de agentes del entorno. También se pretende introducir al alumno en la dirección de la empresa, explorando los factores que determinan la competitividad, los objetivos, el proceso de dirección y de toma de decisiones.

El contenido de la materia se estructura en tres bloques diferenciados que agrupan diez unidades didácticas. El primer bloque Introducción y fundamentos conceptuales, que se divide en dos unidades didácticas, tiene como objetivo aproximar al alumno al concepto de empresa, a su estructura, a su evolución y a su interpretación desde diversos enfoques. El segundo bloque, denominado La dirección de empresas, se estructura en cinco unidades didácticas y su objetivo es comprender el proceso de dirección de la empresa en su conjunto, contextualizando la empresa en el entorno, explorando los fundamentos de la dirección estratégica y analizando la formulación de objetivos y las etapas del proceso de dirección. El último bloque La función de dirección, que está compuesto por tres temas, tiene como objetivo entender la naturaleza y proceso de la función directiva, prestando especial atención a la dirección de las áreas funcionales de la empresa y las implicaciones de la innovación.

2. En las dos asignaturas analizadas se establecen cuatro objetivos generales: a) Adquirir conocimientos básicos: Familiarizarse con los principales conceptos de la dirección y comprender el proceso de dirección; b) Desarrollar competencias directivas activas: Aplicar los conocimientos adquiridos para definir los objetivos, analizar el entorno y la empresa, establecer y seleccionar alternativas de decisión, y ponerlas en práctica; c) Mejorar otras competencias: Mejorar la capacidad de toma de decisiones, de planificación, de comunicación oral y escrita, de trabajo en equipo, de búsqueda y análisis de información y de aprendizaje autónomo; d) Desarrollar actitudes: Espíritu crítico, actitud positiva hacia el cambio, atracción por la toma de decisiones. Sin embargo, estos objetivos se orientan a alcanzar niveles mucho más elevados y tienen un perfil más estratégico y pragmático para la asignatura de quinto curso que para la de primero.

3. Los principales métodos de aprendizaje empleados se agrupan en los cinco siguientes: (1) clases teórico-prácticas; (2) talleres de trabajo; (3) elaboración y exposición de trabajos en equipo; (4) aplicación de mapas conceptuales y (5) tutorías. 


\subsection{Dirección Estratégica de la EMPresa}

Esta asignatura se centra en proporcionar a los alumnos los conocimientos básicos sobre el proceso de dirección estratégica y capacidades directivas para analizar el entorno y la empresa, y diseñar, evaluar y poner en práctica opciones estratégicas. En el plan de estudios actual se sitúa en el primer semestre del quinto curso de la Licenciatura de Administración y Dirección de Empresas. Con esta asignatura se pretende aportar a los alumnos un marco conceptual básico que les permita comprender qué es la dirección estratégica de la empresa, su evolución histórica, sus principales elementos y niveles de análisis; así como el papel que ésta juega en la dirección de la empresa y en las relaciones que mantiene con el resto de agentes de su entorno. El contenido y la metodología empleada en el desarrollo de esta materia se orienta a la mejora de una serie de competencias del alumno como la capacidad de toma de decisiones, de organización y planificación, de comunicación oral y escrita, de trabajo en equipo, de búsqueda y análisis de información, de aprendizaje autónomo, de espíritu crítico, de trabajo en entornos diversos y de actitud estratégica y de cambio. También se pretende que el alumno profundice en las estrategias que determinan la ventaja competitiva empresarial.

El contenido de la materia se estructura en tres bloques diferenciados. El primero Fundamentos de la Dirección Estratégica, que consta de una unidad didáctica que tiene como objetivo aproximar al alumno a la naturaleza y orígenes de la dirección estratégica de la empresa, incidiendo sobre sus elementos básicos, las fases del proceso y sus principales escuelas de pensamiento. El segundo bloque, denominado Análisis Estratégico, se estructura en cinco unidades didácticas y su objetivo es comprender la misión, objetivos y valores de la empresa, el entorno general y específico de la empresa, y el análisis interno de la empresa analizando los recursos y capacidades. El último bloque Formulación de Estrategias de Negocio, que está compuesto por dos unidades didácticas, tiene como objetivo entender la formulación y desarrollo de estrategias de negocio con el fin de adquirir ventajas empresariales en costes y en diferenciación de productos y servicios.

\section{DISEÑO DE LA INVESTIGACIÓN}

El estudio se ha realizado para una población de 87 alumnos de la Licenciatura de Administración y Dirección de Empresas de la Facultad de Ciencias Sociales de Cuenca. De los cuales 59 estaban matriculados en la asignatura de primer curso Introducción a la Dirección de Empresas y 28 en Dirección Estratégica de la Empresa, de quinto curso. La muestra sobre la que hemos realizado los análisis estadísticos está compuesta por 39 alumnos: 19 de IDE y 20 de DEE. Todos ellos cumplimentaron un cuestionario en el que tenían que valorar aspectos tales como el diseño y utilidad de las guías docentes, el nivel de satisfacción con las técnicas docentes utilizadas y el 
desarrollo de las asignaturas, la valoración de la mejora de competencias, el nivel de esfuerzo desarrollado y la satisfacción con los resultados obtenidos.

El cuestionario se estructura en tres bloques: información sobre el plan de aprendizaje, desarrollo de la asignatura y resultados del proceso de formación. En primer lugar, la información sobre el plan de aprendizaje se ha medido con cuatro escalas: claridad de información del profesor, compuesta por 8 ítems; medios utilizados para consultar la guía, formada por 5 ítems; valoración de la guía, medida con 5 ítems; e información sobe el sistema de evaluación con 6 ítems. En el segundo bloque, correspondiente al desarrollo de la asignatura, se incluyen tres escalas: la satisfacción con el proceso de formación, que se mide con 11 ítems, las técnicas de aprendizaje activo utilizadas en la asignatura, medidas fon 9 ítems y el nivel de satisfacción con las técnicas de aprendizajes utilizadas, escala compuesta por 9 ítems. El tercer bloque hace referencia a los resultados obtenidos del proceso de formativo y, en este sentido, se valoran la mejora de las competencias en los alumnos, medidas a través de 11 ítems y la satisfacción con el resultado del aprendizaje y con el sistema de evaluación continua, cada una de estas ultimas variables se ha valorado con un ítem.

Las variables relativas a la claridad con que informa el profesor, valoración de la guía docente, satisfacción con la asignatura, nivel de esfuerzo, mejora de las competencias y satisfacción del resultado y de la evaluación se han medido con escala Likert de cinco puntos. Mientras que los ítems referidos a los medios utilizados para consultar la guía, información sobre el sistema de evaluación y técnicas de aprendizaje utilizadas en la asignatura se han medido con una escala dicotómica. Además, al final del cuestionario se incluyeron tres preguntas abiertas para recoger información acerca de los aspectos que mas les han gustado de la asignatura, los que menos les han gustado y cualquier sugerencia sobre el desarrollo y/o evaluación de la asignatura.

Una vez recogida la información, pasamos al tratamiento de la misma a través de los siguientes análisis estadísticos. En primer lugar, hallamos los valores medios de todas las variables para cada asignatura y también la media muestral. Posteriormente, realizamos, con las variables continuas, un análisis de la varianza univariante ANOVA con el fin de comparar las diferencias de medias entre grupos con el estadístico F. Por otro lado, planteamos una tabulación cruzada para analizar las frecuencias de las variables discretas, analizando la asociación existente entre variables mediante la prueba Chi-cuadrado de Pearson (Hair, Anderson, Tatham y Black, 1999).

\section{RESULTADOS}

En este apartado presentamos los resultados obtenidos de los análisis estadísticos realizados de comparación entre grupos que nos muestran algunas diferencias entre los alumnos que cursan la asignatura de IDE (primer curso) y los de DEE 
(quinto curso). La información recogida y analizada estadísticamente se ha estructurado en tres bloques: a) información sobre el plan de aprendizaje, b) desarrollo de la asignatura y c) resultados del proceso de formación.

\subsection{Información sobre el Plan de Aprendizaje}

Dentro del primer bloque -ver tabla1-, referido a la información que se aporta sobre el plan, los datos ponen de manifiesto que los alumnos valoran muy positivamente la claridad de información del profesor sobre el plan de aprendizaje, puesto que la mayoría de los ítems tienen valores por encima de 3,5 -en una escala de cinco-, destacando que son algo superiores los valoraciones realizadas por los estudiantes de DEE. Esto significa que los alumnos del último curso interpretan mejor los nuevos métodos de enseñanza aprendizaje; sobre todo los objetivos del aprendizaje, los materiales de apoyo, la organización temporal de la asignatura. No obstante, el ítem que mide el desarrollo de las clases presenta un valor medio superior en el grupo de primer curso, esto se puede deber a que están más familiarizados los alumnos con esta técnica.

En relación a los medios de consulta de la guía docente utilizados por los estudiantes, lo más destacable es el predominio de los medios informáticos, tanto en los alumnos de primero como en los de quinto. Aunque debemos matizar que los estudiantes de DEE no han utilizado el CD con la guía porque esta asignatura al ser de quinto curso no está incluida en el proyecto piloto de la Facultad, que ha sido el marco en el que se han elaborado las guías docentes en este tipo de soporte, distribuidas sólo entre los alumnos de las asignaturas de primer curso. Los estudiantes de ambas asignaturas, utilizan más la plataforma virtual interna de la Universidad, denominada Red-Campus, que el tradicional servicio de reprografía $-97,4 \%$ para el conjunto de la muestra-. Si bien el elemento clave de información que prevalece por encima de cualquier otro medio es la presentación y explicación de la guía docente en clase por parte del profesor al inicio del cuatrimestre $-100 \%$ en ambos casos-. Cabe destacar en este apartado que, la guía es considerada como un recurso básico para un desarrollo eficaz del plan de aprendizaje principalmente por los alumnos de quinto curso $-73,7 \%$-, en este caso concreto el test Chi-cuadrado es significativo al $95 \%$.

Por otra parte, los resultados del análisis de la varianza muestran que existen diferencias significativas en todos los ítems referidos a la valoración de la guia por parte de los alumnos, excepto en el contenido de la guía, que se valora de forma muy parecida en ambas asignaturas. Los alumnos de primer curso destacan la claridad y el contenido $-7,57$ sobre 10 en ambos casos-, mientras que los de quinto dan mayor valor a la claridad -8,26y utilidad de la guía -8,21-. Además, en todos los ítems, la valoración media de los alumnos de último curso es mayor que la otorgada por los alumnos de primero.

En cuanto a la percepción que los alumnos tienen acerca de la información sobre la evaluación, destacamos que en todos los ítems hay valores muy elevados, 
con frecuencias por encima del $90 \%$ en la mayoría de los casos, lo que significa que se consideran muy bien informados en todas las cuestiones relacionadas con el sistema de evaluación. En este sentido, los datos estadísticos revelan que no existen diferencias significativas entre las dos asignaturas. No obstante, podemos señalar que los alumnos de quinto curso han asignado el valor más bajo a los procedimientos de retroalimentación $-77,8 \%$ - Los de primer curso consideran que la explicación más débil ha sido la realizada sobre el proceso de retroalimentación -84,2\%-y el peso que tiene cada componente en el sistema de evaluación -85\%.

TABLA 1. INFORMACIÓN SOBRE EL PLAN DE APRENDIZAJE

\begin{tabular}{|c|c|c|c|c|c|c|}
\hline & & $\mathrm{mF}^{\prime}$ & DFF & MEDIA & \multicolumn{2}{|c|}{ ANOVA } \\
\hline & & Escala 1-5 & Escala 1-5 & Escala 1-5 & $\mathbf{F}$ & Sig. \\
\hline \multirow{8}{*}{ 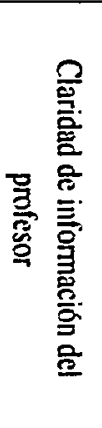 } & Objetivos del aprendizaje & 3.89 & 4.10 & 4.00 & 2.717 & 0.108 \\
\hline & Materiales de apoyo & 3,47 & 3.63 & 3.55 & 0.764 & 0,388 \\
\hline & Desarrollo de las clases & 4.21 & 3.78 & 4,00 & 3,310 & 0,077 \\
\hline & Bibliografía básica & 3.31 & 3,78 & 3,55 & 4.445 & 0.042 \\
\hline & Bibliografía complementaria & 2,89 & 3.21 & 3.05 & 2.634 & 0.113 \\
\hline & Organización temporal asign. & 3.73 & 4.00 & 3,86 & 1.731 & 0,197 \\
\hline & Métodos docentes & 3.72 & 3.84 & 3.78 & 0.256 & 0,616 \\
\hline & Sistemas de evaluación & 3,77 & 3.78 & 3.78 & 0.002 & 0,963 \\
\hline & & Porcentaje & Porcentaje & Porcentaje & Chi-cuadrado & Sig. \\
\hline \multirow{6}{*}{ 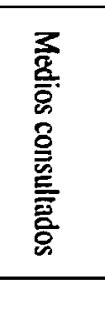 } & En el CD del Centro & 31.6 & $\overline{0}$ & 16.7 & 6.442 & 0.011 \\
\hline & Red-Campus & 100 & 94.7 & 97.4 & 1,080 & 0.299 \\
\hline & Reprografía & 77.8 & 63.2 & 70.3 & 0,946 & 0,331 \\
\hline & Presentado en clase & 100 & 100 & 100 & - & - \\
\hline & La guía es un recurso básico & 36.8 & 73.7 & 55.3 & 5,216 & 0,022 \\
\hline & & Escala 1-10 & Escala 1-10 & Escala 1-10 & $\mathbf{F}$ & Sig. \\
\hline \multirow{6}{*}{ 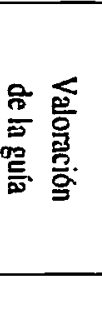 } & Claridad & 7,57 & 8.26 & 7.92 & 3.971 & 0.054 \\
\hline & Comprensión & 6,89 & 7.78 & 7.34 & 5.594 & 0.024 \\
\hline & Lenguaje & 7,10 & 7.94 & 7.52 & 5.953 & 0.020 \\
\hline & Contenido & 7.57 & 7,94 & 7.76 & 1,018 & 0.320 \\
\hline & Utilidad & 7.05 & 8.21 & 7.63 & 3,818 & 0,059 \\
\hline & & Porcentaje & Porcentaje & Porcentaje & Chi-cuadrado & Sig. \\
\hline \multirow{6}{*}{ 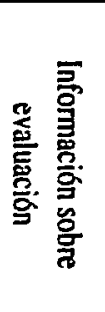 } & Procedimientos de evaluación & 100 & 100 & 100 & - & - \\
\hline & Momento de la evaluación & 89,5 & 89.5 & 89.5 & 0,000 & 1,000 \\
\hline & Criterios de evaluacion & 100 & 94.7 & 97.4 & 1.027 & 0.311 \\
\hline & Componentes de la evaluación & 100 & 94.7 & 97.4 & 1.027 & 0.311 \\
\hline & Peso de cada componente & 85 & 94.7 & 89.7 & 1.004 & 0.316 \\
\hline & Procedto. de retroalimentación & 84.2 & 77.8 & 81.1 & 0.249 & 0,618 \\
\hline
\end{tabular}




\subsection{Desarrollo de la Asignatura}

En el segundo bloque se analiza la implantación del plan de aprendizaje y el desarrollo de la asignatura -tabla 2-. En primer lugar abordamos la satisfacción de los alumnos con el proceso de formación. Los datos obtenidos de los análisis estadísticos revelan que, de manera general, los estudiantes manifiestan un elevado grado de satisfacción con la implantación de esta nueva metodología. Los alumnos de DEE en quinto curso están satisfechos principalmente con la localización del profesor $-4,15$ sobre 5 - y con los objetivos de la asignatura 4,05-. Los alumnos de primer curso muestran mayor satisfacción que los de quinto en cuestiones relacionadas con el sistema tradicional de enseñanza como, por ejemplo, en el desarrollo de las clases presenciales -4,15-, así como en la valoración general de la asignatura $-4,15-$.

En relación a las técnicas de aprendizaje empleadas para impartir la asignatura, los datos ponen de manifiesto que en las dos asignaturas predominan las clases presenciales y el trabajo en grupo -en ambos casos el 100\%-, seguidas de la resolución de casos y ejercicios prácticos $-97,3 \%$ y $94,7 \%$ respectivamente. Debemos señalar, asimismo, que los estudiantes de quinto curso asisten más, por término medio, a conferencia externas $-33 \%-$, celebradas fuera del plan de estudios de la titulación, con el fin de ampliar conocimientos; mientras que los alumnos de primero participan más que los de quinto en seminarios de especialización organizados dentro de la propia asignatura para profundizar en los contenidos teóricos $-26,3 \%$ -

Los datos acerca del nivel de satisfacción con las actividades de aprendizaje desarrolladas ponen de manifiesto que los alumnos de primero se sienten muy satisfechos con el desarrollo de las clases presenciales -4,00-, así como con la realización de casos y problemas -4,05- Mientras que los estudiantes de quinto curso manifiestan mayor satisfacción con la elaboración de trabajos en grupo $-4,21-$, seguidos de la asistencia a conferencias $-3,85-$, realización de prácticas 3,83-, así como análisis críticos de documentos -3.78-.

Una cuestión clave en el desarrollo de la asignatura es la valoración que los estudiantes hacen del esfuerzo realizado en la asignatura, los alumnos de quinto curso asumen un esfuerzo mayor que los de primero $-4,42$ y 3,40 respectivamente-. Además, apreciamos que la diferencia en este ítem es estadísticamente significativa. 
Tabla 2. Desarrollo de la asignatura

\begin{tabular}{|c|c|c|c|c|c|c|}
\hline & & IDE & DEE & $\begin{array}{c}\text { MEDIA } \\
\text { poblacional }\end{array}$ & & \\
\hline & & Escala 1-5 & Escala 1-5 & Escala 1-5 & $\mathbf{F}$ & Sig. \\
\hline \multirow{12}{*}{ 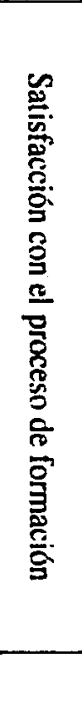 } & Objetivos de la asignatura & 4.00 & 4,05 & 4,02 & 0,136 & 0,714 \\
\hline & Materiales de apoyo & 3.68 & 3,68 & 3,68 & 0,000 & 1,000 \\
\hline & Desarrollo de las clases & 4.15 & 3.78 & 3,97 & 3,392 & 0,074 \\
\hline & Bibliografia básica & 3.47 & 3,84 & 3.65 & 3,500 & 0.070 \\
\hline & Bibliografía complementaria & 3,21 & 3.26 & 3,23 & 0.050 & 0,824 \\
\hline & Organización temporal asignatura & 4,60 & 3,78 & 3.89 & 1.152 & 0,290 \\
\hline & Métodos docentes & 3,89 & 3,63 & 3,76 & 1,301 & 0,262 \\
\hline & Sistemas de evaluación & 4.00 & 3.73 & 3,86 & 2,313 & 0,137 \\
\hline & Horario de tutorías & 3.36 & 3,63 & 3,50 & 1,844 & 0,183 \\
\hline & Localización del profesor & 3,52 & 4.15 & 3.84 & 10,286 & 0.003 \\
\hline & Valoración general de la asignatura & 4,15 & 3,94 & 4,05 & 1,600 & 0,214 \\
\hline & & Porcentaje & Porcentaje & Porcentaje & $\begin{array}{c}\text { Chi- } \\
\text { cuadrado }\end{array}$ & Sig. \\
\hline \multirow{10}{*}{ 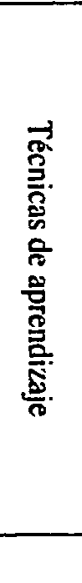 } & Clases presenciales & 100 & 100 & 100 & - & - \\
\hline & Trabajo en grupo & 100 & 100 & 100 & - & - \\
\hline & Casos - problemas & 100 & 94,4 & 97,3 & 1,085 & 0,298 \\
\hline & Prácticas & 94.7 & 94,7 & 94,7 & 0,000 & 1,000 \\
\hline & Búsqueda documentales & 72.2 & 68.4 & 70.3 & 0,064 & 0,800 \\
\hline & Comentarios de texto & 100 & 77,8 & 89.2 & 4.734 & 0.030 \\
\hline & Análisis crítico documentos & 88,9 & 82.4 & 85.7 & 0,305 & 0,581 \\
\hline & Conferencias & 21.1 & 33,3 & 27,0 & 0,707 & 0,401 \\
\hline & Seminarios & 26.3 & 12.5 & 20,0 & 1,036 & 0,309 \\
\hline & & Escala 1-5 & Escala 1-5 & Escala 1-5 & $\mathbf{F}$ & Sig. \\
\hline \multirow{10}{*}{ 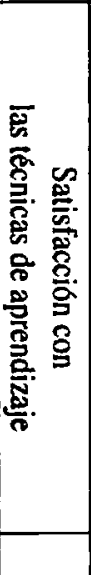 } & Clases presenciales & 4,00 & 3,73 & 3,86 & 1,339 & 0.255 \\
\hline & Trabajo en grupo & 3.94 & 4,21 & 4,07 & 1,679 & 0,203 \\
\hline & Casos - problemas & 4,05 & 3,43 & 3,77 & 5,743 & 0,022 \\
\hline & Prácticas & 3,83 & 3,83 & 3,83 & 0,000 & 1,000 \\
\hline & Búsqueda documentales & 3,58 & 3,38 & 3.50 & 0.648 & 0428 \\
\hline & Comentarios de texto & 4,05 & 3.53 & 3,82 & 8,333 & 0,007 \\
\hline & Análisis crítico documentos & 3,66 & 3.78 & 3,71 & 0,205 & 0,654 \\
\hline & Conferencias & 2,92 & 3.85 & 3.23 & 20,195 & 0,000 \\
\hline & Seminarios & 3,07 & 3,66 & 3,17 & 2.347 & 0,146 \\
\hline & Esfuerzo realizado asignatura & 3,40 & 4,42 & 3,89 & $21_{\infty} 562$ & 0,000 \\
\hline
\end{tabular}


En relación a la estimación del tiempo empleado por el alumno para el desarrollo del total de actividades - tabla 3-, observamos que en ambos casos les dan una sobre valoración a la variable tiempo. Partiendo de que los estudiantes deben realizan todas las tareas en 150 horas, puesto que se tratan de asignaturas de 6 créditos, los alumnos de primer curso, de media, emplean 174,90 horas, mientras que los de quinto curso necesitan 237,15 horas para el desarrollo de la asignatura. En ambos casos, por tanto, se supera el tiempo máximo necesario según los criterios de aplicación de los créditos ECTS ${ }^{4}$.

Tabla 3. Estimación del tiempo empleado (en el cuatrimestre)

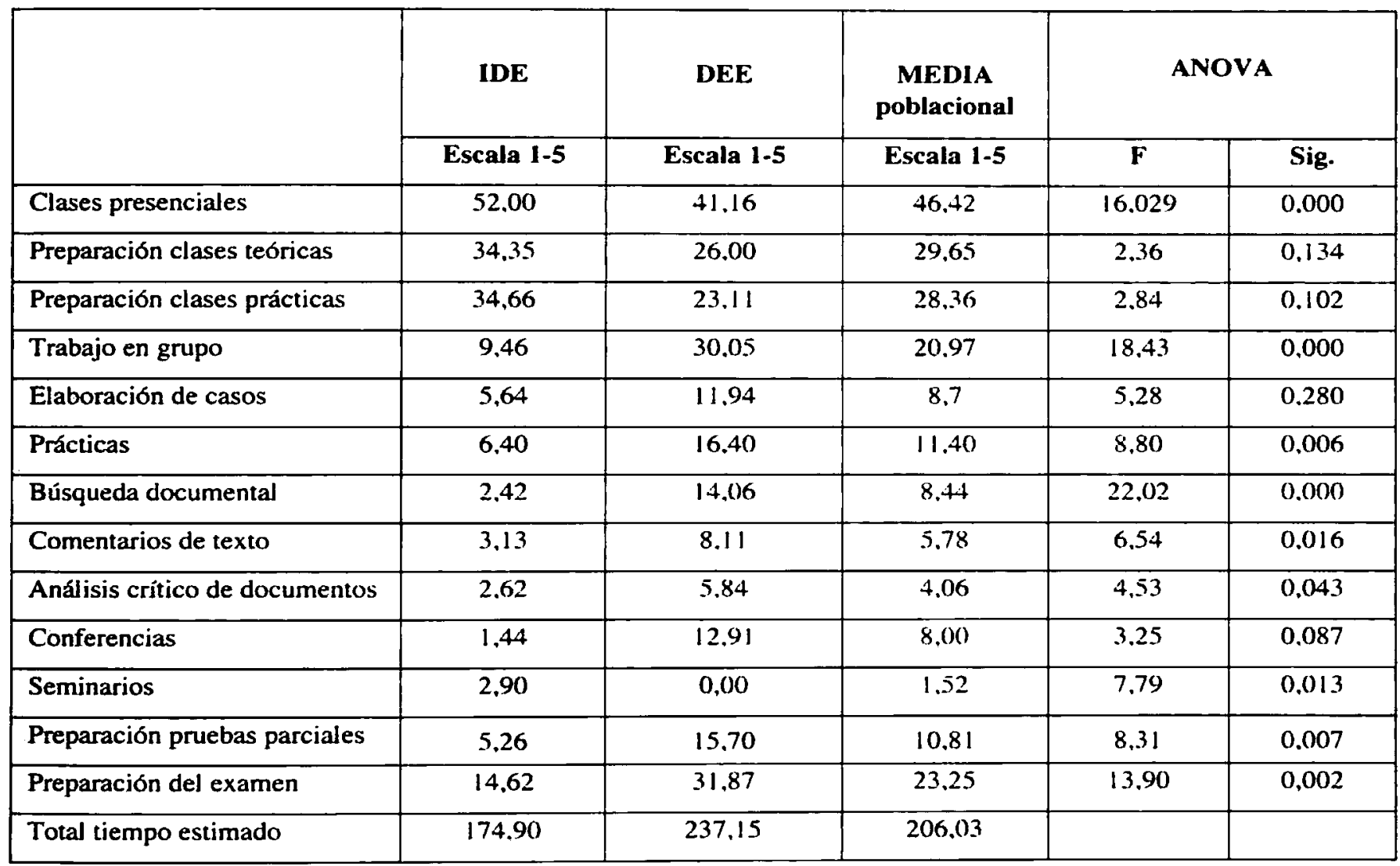

Además, se observan algunas diferencias entre los alumnos de las dos asignaturas; por ejemplo, a las clases presenciales les dedican más tiempo los alumnos de primero que los de quinto -52 horas y 41,16 horas respectivamente-, de igual modo ocurre con la preparación y repaso de los contenidos impartidos en clase, tanto los

4. Debemos señalar que comprobamos un cierto sesgo en la percepción subjetiva del tiempo dedicado, que se pone de manifiesto en que los alumnos -especialmente los de primer curso- indican una dedicación a las clases presenciales muy superior a las reales. 
teóricos como los prácticos. Por su parte, los alumnos de quinto dedican más tiempo a actividades como elaboración de casos $-11,94$ horas-, resolución de ejercicios prácticos $-16,40$ horas-, búsqueda de información $-14,06$ horas-, asistencia a conferencias $-12,91 \%-$, preparación de pruebas parciales $-15,70$ horas- y examen final $-31,87$ horas. Comprobamos que la diferencia más importante se advierte en el tiempo que emplean en realizar trabajos en grupo, en que los estudiantes de quinto curso dedican aproximadamente tres veces más de tiempo en esta actividad que los de primero.

\subsection{Resultados del Proceso de Formación}

En el tercer bloque analizamos los resultados del proceso de formación, y dentro del él, la mejora de las competencias genéricas de los alumnos -tabla 4-. Los datos estadísticos ponen de manifiesto que los alumnos de quinto curso perciben en mayor medida que los de primero una mejora en sus competencias, sobre todo en la habilidad para buscar información $-4,26$ por los de quinto-, capacidad de trabajo en grupo $-4,21$, adaptación a nuevas situaciones $-4,05-$, capacidad de análisis y síntesis $-4,00-$, capacidad de organización y planificación -4,00-, capacidad para la toma de decisiones $-4,00$.

TABLA 4. Resultados Del proceso de formación

\begin{tabular}{|c|c|c|c|c|c|c|}
\hline & & IDE & DEE & MEDIA & \multicolumn{2}{|c|}{ ANOVA } \\
\hline & & Escala 1.5 & Escala 1-5 & Escala 1-5 & $\mathbf{F}$ & Sig. \\
\hline \multirow{13}{*}{ 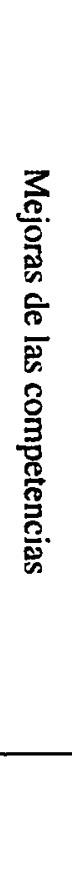 } & Capacidad de análisis y síntesis & 3.78 & 4,00 & 3,89 & 2,118 & 0,154 \\
\hline & Capacidad organización y planificación. & 3,78 & 4,00 & 3,89 & 0,883 & 0,354 \\
\hline & Habilidad buscar información & 3,78 & 4.26 & 4.02 & 4,556 & 0,040 \\
\hline & Capacidad resolución de problemas & 3,47 & 3,94 & 3,71 & 3,898 & 0,056 \\
\hline & Capacidad de tomar decisiones & 3,52 & 4,00 & 3,76 & 3,375 & 0,074 \\
\hline & Capacidad de autocrítica & 3,73 & 3,84 & 3,78 & 0,234 & 0,632 \\
\hline & Compromiso ético en el trabajo & 3,68 & 3,89 & 3,78 & 0,847 & 0,364 \\
\hline & Aprendizaje autónomo & 3,78 & 3,94 & 3,86 & 0,704 & 0,407 \\
\hline & Capacidad de trabajo en grupo & 3.84 & 4,21 & 4,02 & 3,973 & 0,054 \\
\hline & Adaptación a nuevas situaciones & 3,84 & 4,05 & 3,94 & 1,125 & 0,296 \\
\hline & Comprensión del contenido de asignatura & 4,05 & 4.05 & 4,05 & 0,000 & 1,000 \\
\hline & Satisfacción con resultado aprendizaje & 3,61 & 3,68 & 3,64 & 0,071 & 0,792 \\
\hline & Satisfacción con resultado evalu. continua & 2,27 & 3,16 & 2,82 & 5,136 & 0,032 \\
\hline
\end{tabular}


En relación con los resultados obtenidos del proceso formativo, también analizamos la satisfacción con el resultado y los datos revelan que los alumnos de quinto curso muestran mayor nivel de satisfacción que los de primero, esto es así, tanto en relación con el resultado del aprendizaje -3,68- como con el resultado de la evaluación continua -3,16-. En este último caso, los alumnos de primer curso se sitúan por debajo de la puntuación media de la escala -2,27-.

\section{CONCLUSIONES}

Los análisis estadísticos realizados nos han permitido identificar los perfiles de los alumnos de primer y último curso de Administración y Dirección de Empresas, que son diferentes en la forma de percibir y afrontar la implantación de los créditos europeos ECTS, así como en la satisfacción con los resultados obtenidos con este nuevo plan de aprendizaje.

La mayoría de investigaciones en este campo se centran generalmente en el estudio de una experiencia concreta en un determinado curso o incluso en toda una titulación; sin embargo, en este caso, nuestro trabajo ha consistido en un análisis comparativo entre alumnos de primer y último curso. De este modo contribuimos a analizar la reacción de los estudiantes de primer curso cuando todavía no conocen los nuevos métodos de aprendizaje activo y el sistema de créditos ECTS y compararla con la percepción y aceptación de los alumnos de quinto curso, que están más familiarizados con el sistema de enseñanza aprendizaje, puesto que ellos sí han tenido anteriormente experiencias puntuales en estas metodologías y que tienen la perspectiva de una salida próxima al mercado laboral.

Las diferencias en los perfiles de los alumnos de IDE y DEE nos permiten extraer algunas conclusiones que contribuyan a orientar la implantación de los créditos ECTS en los primeros y últimos cursos de cualquier titulación, con el fin de mejorar la aplicación de nuevas técnicas de aprendizaje y obtener mejores resultados.

Los alumnos que cursan la asignatura de DEE de quinto curso se caracterizan principalmente porque valoran más positivamente el aprendizaje de competencias, sobre todo aquéllas relacionadas con el desarrollo futuro de su trabajo como la capacidad para trabajar en grupo, de toma de decisiones, de búsqueda de información y de adaptación a nuevas situaciones, entre otras. Estos alumnos perciben que el nivel de esfuerzo exigido para el desarrollo de este nuevo proceso de formación es muy elevado; sin embargo, y a pesar del importante esfuerzo que les supone esta nueva metodología, manifiestan un grado bastante elevado de satisfacción con los resultados obtenidos. Por otro lado, aunque 
valoran muy positivamente la implantación y el desarrollo del plan de aprendizaje, muestran una actitud más crítica sobre el proceso de aprendizaje en general, básicamente con la organización temporal, el sistema de evaluación y la impartición de las clases presenciales y resolución de casos y problemas. Por otra, parte y en relación a la utilidad de la guía, no sólo ponen de manifiesto la gran utilidad que supone disponer de la guía docente de la asignatura, sino que ellos realmente le dan un uso importante como herramienta clave de ayuda al aprendizaje. Estos alumnos, dado que se encuentran en el último curso de la carrera poseen una visión global del funcionamiento del sistema educativo universitario en general y, además, la mayoría de ellos han desarrollado con anterioridad otras experiencias en el marco de los ECTS. Este hecho implica que estos estudiantes comprendan en mayor medida la metodología empleada para el desarrollo del plan de aprendizaje, los objetivos, así como el sistema de técnicas y actividades, en su conjunto, aplicadas en la impartición de la asignatura. Estos alumnos, dado que comprenden mejor el desarrollo del plan de formación, utilizan de manera más eficiente la guía docente y la consideran un recurso básico de trabajo.

Frente a este tipo de estudiantes de último curso se encuentran los alumnos de la asignatura IDE de primer curso. Éstos muestran un perfil diferente. En primer lugar, los alumnos de primero valoran en mayor medida el desarrollo de aquéllas competencias vinculadas con la comprensión de conceptos y contenidos teóricos de la asignatura. Este hecho puede ser debido a que mantienen la inercia a los objetivos y metodología de la enseñanza en secundaria y todavía no comprenden la utilidad que les pueden aportar las metodologías de aprendizaje activo para el desarrollo de nuevas competencias. Otra diferencia significativa de estos estudiantes es que les ha supuesto un nivel de esfuerzo menor de lo que manifiestan los de quinto; sin embargo, a su vez, muestran un grado de satisfacción menor, tanto en el aprendizaje en general como en el sistema de evaluación del proceso.

En relación al tiempo empleado para el desarrollo de la asignatura, en primer lugar, se advierte que en ambos casos los alumnos han realizado una estimación subjetiva, sobrevalorando el tiempo empleado, que supera el considerado como máximo en la normativa de Bolonia. La principal diferencia entre los alumnos de las dos asignaturas es que los alumnos de primer curso dedican más tiempo a las tareas, dentro de la nueva metodología, que más se asemejan al sistema de aprendizaje tradicional: clases presenciales y preparación y repaso de esas clases. Mientras que los alumnos de quinto curso emplean más tiempo en el desarrollo y realización de actividades de aprendizaje activo, directamente relacionadas con la implantación de los nuevos créditos europeos ECTS, como por ejemplo trabajos en grupo, elaboración de casos y ejercicios prácticos, búsqueda documental, etc. 
En suma, destacamos que los alumnos de quinto interpretan mejor y captan de una manera más eficaz la nueva metodología de aprendizaje activo utilizada para implantar los créditos ECTS, puesto que perciben un mayor desarrollo y mejora de sus competencias al final del proceso de aprendizaje. Sin embargo, también muestran un espíritu más crítico con este nuevo sistema, sobre todo, por la elevada carga de trabajo que les supone; aunque lo aceptan porque revelan un elevado nivel de satisfacción con los resultados obteridos.

Finalmente, a partir de los resultados obtenidos, de la revisión de las respuestas de los alumnos a las preguntas abiertas y de la experiencia desarrollada en los últimos años, los profesores que hemos participado en este proyecto planteamos una serie de recomendaciones para la implantación de ECTS. Las líneas de trabajo que proponemos para que la aplicación de ECTS sea más eficaz y eficiente en las siguientes: a) se requiere un elevado nivel de explicación y motivación a los alumnos de último curso y una planificación y control adecuados para los alumnos de primer curso; b) es conveniente incidir y evaluar la mejora de determinadas competencias, como la toma de decisiones, el trabajo en grupo, la búsqueda y análisis de información y la comprensión global de los proceso de dirección; c) se deben planificar detalladamente las actividades y controlar el tiempo de dedicación de los alumnos y profesores a las actividades del plan de aprendizaje -la elaboración de la guía docente puede ser de mucha ayuda-; d) es recomendable incorporar progresivamente nuevos sistemas de evaluación incorporando criterios de eficiencia. eficacia y confianza, mejorando el retorno progresivo de información sobre evaluación del proceso de aprendizaje a los alumnos; e) se necesita una amplia formación de los profesores sobre diversos métodos de aprendizaje y evaluación; f) es aconsejable aplicar el nuevos sistema de aprendizaje en primer lugar a materias con un marcado carácter práctico y escasos alumnos; g) una forma de facilitar el proceso y evitar conflictos es realizar los planes de entrenamiento en el marco de una acción coordinada con un grupo de docencia; y h) se debe establecer un proceso continuo de revisión del sistema de aprendizaje que permita incorporar las mejoras sugeridas por los alumnos y la reflexión individual y colectiva sobre experiencias anteriores.

Debemos asumir las limitaciones de este trabajo, derivadas del carácter específico del plan de entrenamiento recogido en la investigación sobre dos asignaturas concretas -IDE y DEE-. Sin embargo, consideramos que la experiencia desarrollada durante varios años y ampliada a otras materias de diversas titulaciones -Relaciones Laborales, Arquitectura Técnica, Humanidades, etc.- nos ha permitido realizar un análisis más profundo de los aspectos analizados en el trabajo. 


\section{BIBLIOGRAFÍA}

BLOOM B. S. (ed.) (1956) Taxonomy of Educational Objectives, the classification of educational goals - Handbook I: Cognitive Domain New York: McKay.

DECLARACIÓN DE LA SORBONA (1998): Declaración conjunta para la armonización del diseño del Sistema de Educación Superior Europeo.

DECLARACIÓN DE BOLONIA (1999): Declaración conjunta de los Ministros Europeos de Educación.

HAIR, J.; ANDERSON, R.; TATHAM, R.; BLACK, W. (1999): Análisis Multivariante, $5^{\text {a }}$ ed., Prentice-Hall Iberia, Madrid.

MECD (2003): Documento marco "La integración del sistema español en el espacio europeo de educación en el espacio europeo de Enseñanza Superior", Ministerio de Educación y Deporte.

OCDE (2000): "Knowledge Management in the Leaming Society", Paris: Center for Educational Research Innovation.

STEWART, D. (1997): "African urbanization: Dependent linkages in a global economy", Journal for Economic and Social Geograpby, $\mathrm{n}^{0}$ 88, págs.251-61.

\section{ANEXO 1: EVALUACIÓN DEL APRENDIZAJE}

La evaluación del aprendizaje de los alumnos tendrá dos orientaciones complementarias, que permitirán comprobar la consecución de los objetivos planteados. Por un lado, se realizará una evaluación continua, controlando el esfuerzo y los progresos del alumno en una serie de competencias y destrezas planteadas en los objetivos de la materia. Por otro lado, se realiza una prueba test intermedia y una prueba teórico-práctica final más orientada a comprobar el dominio de los conceptos básicos de la asignatura, las principales relaciones entre los mismos y su aplicación a casos especificos de empresa. El sistema de evaluación descrito se establece como opción $A$, y la calificación final viene determinada por los siguientes criterios

- Opción A (Evaluación continua):

- Elaboración y exposición de casos realizados en equipo. Su valoración es de un $15 \%$ de la nota final. Se tendrán en cuenta los siguientes criterios: elección de la empre$\mathrm{sa}$, adecuación del caso a la teoría, profundidad del análisis de la empresa, claridad en la exposición, presentación de transparencias, diversidad y claridad de bibliografia y material, conclusiones, creatividad, formato escrito (índice, estructura, etc.), valoración 
de los compañeros, etc. Se aplicará una corrección en la valoración de cada uno de los miembros de un grupo en función de su contribución individual al trabajo.

- Prueba test intermedia (entre 10 y 20 preguntas). Su valoración es de un $10 \%$ y no permite eliminar materia.

- Participación activa en clase. Su valoración es de un 20\%. Esta nota está configurada por numerosos ítemes que están relacionados con resolución de casos, análisis crítico de casos breves, elaboración y exposición de casos, resolución de preguntas de reflexión, toma de decisiones para el diseño de estrategias, valoración de casos de otros compañeros, intervenciones y preguntas. Los ítemes tendrán una ponderación diferente en función del grado de esfuerzo realizado en cada caso (ej. Para el mismo grado de dificultad, tienen mayor ponderación los trabajos individuales que los colectivos).

- Participación en otras actividades complementarias (valoración de 5\%). Estas actividades pueden ser seminarios realizados fuera de la asignatura, visitas a empresas, jornadas, formación en idiomas, formación en informática, prácticas en empresas, etc. Para la valoración de este apartado, es necesario que se justifique la utilidad de la realización de la actividad para alcanzar los objetivos de la empresa y que se aporten los documentos acreditativos de la actividad.

- Examen final teórico-práctico. Supone el $50 \%$ de la nota final. Está formado por diversas cuestiones de carácter teórico o práctico. Para superar esta prueba se debe obtener, al menos una nota de 3,5/10.

- Opción B (Evaluación tradicional):

- Examen test de 25 preguntas (cada tres incorrectas descontará una correcta y se deberá obtener una puntuación mínima de 12) + Examen final teórico-práctico. Está formado por varias cuestiones de carácter teórico o práctico. Es necesario superar ambas partes por separado y la calificación de la asignatura corresponderá en la obtenida en el teórico-práctico. Supone un $90 \%$ de la nota final.

- Presentación individual por escrito de un caso de empresa adaptado a un punto de la asignatura. Su valoración es de un $10 \%$ de la nota final. Será un trabajo individual asignado por los profesores de la asignatura y para ser valorado deberá ser presentado antes del examen. Se valorará siguiendo los siguientes criterios: elección de la empresa, adecuación a teoría, profundidad análisis empresa, bibliografía y material, conclusiones, creatividad, formato escrito - carátula, índice estructura,...-).

Previamente a la realización de la prueba final, se publicará una lista con las notas correspondientes a la evaluación continua, para que los alumnos puedan decidir a que opción presentarse del examen final. Los alumnos que pasen a la opción $\mathrm{B}$ podrán sustituir la entrega de un trabajo sobre un caso de una empresa/sector por la nota obtenida en la elaboración y exposición de casos. En caso de suspender con la opción A en la convocatoria ordinaria, la nota obtenida por los diferentes apartados de la evaluación continua se mantendrá hasta la siguiente convocatoria. 